University of Rhode Island

DigitalCommons@URI

Mechanical, Industrial \& Systems Engineering

Faculty Publications

2015

\title{
Casimir effect modified surface energy of a nanocavity in homogeneous media
}

\author{
Yi Zheng \\ University of Rhode Island, zheng@uri.edu \\ Arvind Narayanaswamy
}

Follow this and additional works at: https://digitalcommons.uri.edu/mcise_facpubs

The University of Rhode Island Faculty have made this article openly available.

Please let us know how Open Access to this research benefits you.

This is a pre-publication author manuscript of the final, published article.

Terms of Use

This article is made available under the terms and conditions applicable towards Open Access Policy Articles, as set forth in our Terms of Use.

\section{Citation/Publisher Attribution}

Zheng, Y. and Narayanaswamy, A. (2015), Casimir effect modified surface energy of a nanocavity in homogeneous media. ANNALEN DER PHYSIK, 527: 499-506. doi: 10.1002/andp.201500145

Available at: http://dx.doi.org/10.1002/andp.201500145

This Article is brought to you for free and open access by the Mechanical, Industrial \& Systems Engineering at DigitalCommons@URI. It has been accepted for inclusion in Mechanical, Industrial \& Systems Engineering Faculty Publications by an authorized administrator of DigitalCommons@URI. For more information, please contact digitalcommons-group@uri.edu. 


\title{
Casimir effect modified surface energy of a nanocavity in homogeneous media
}

\author{
Yi Zheng* \\ Department of Mechanical, Industrial and Systems Engineering, \\ University of Rhode Island, Kingston, RI 02881, USA
}

Arvind Narayanaswamy ${ }^{\dagger}$

Department of Mechanical Engineering, Columbia University, New York, NY 10027, USA

\begin{abstract}
We determine the regularized van der Waals contribution to pressure within a spherical cavity of vapor in a homogeneous, isotropic, infinite medium. The spherical Hamaker function, $A_{s}$, has been defined, for the first time, in contrast to the conventional Hamaker function for planar surfaces, $A_{h}$. For the materials under consideration, the pressure inside the cavity varies as $A_{s} / 6 \pi a^{3}$, where $a$ is the radius of the cavity. For radii below a transition radius, the surface energy (or surface tension) becomes size dependent and could have important implications for homogeneous nucleation of nanosized bubbles in liquids, as well as cavitation of bubbles.
\end{abstract}

\section{INTRODUCTION}

Dispersion forces, such as van der Waals force and Casimir force, arise due to the modification in the fluctuations of the electromagnetic field by the presence of boundaries. Current understanding of van der Waals force (we will use the term van der Waals to include Casimir forces too) between macroscopic objects is based on Lifshitz theory. Lifshitz [1] used Rytov's theory of fluctuational electrodynamics [2] to determine the force between two semi-infinite half-spaces separated by a vacuum gap (a planar cavity). Recent works have shown that the generalization of Lifshitz theory can be applied not only to the multilayered media [3-9], but also to the objects of arbitrary shapes [10], such as ellipsoids [11, 12], cylinders $[13,14]$ and cubes $[10,15]$. In this work, we find the van der Waals contribution to pressure within a cavity of vapor, the electromagnetic properties of which is assumed to be identical with that of vacuum, in a homogeneous, isotropic, infinite liquid (see Fig. 1).

The earliest work on the fluctuational contribution to pressure within a sphere is that of Boyer, who, inspired by Casimir's model for a charged particle, determined the forces on an uncharged conducting spherical shell [16]. Milton et al. [17] also studied the same problem using the source theory formalism of Schwinger et al. [18] and reached the same conclusion as Boyer that the self stress on the sphere is repulsive. Subsequently, Milton [19] and Milton and $\mathrm{Ng}$ [20] also determined the van der Waals self-stress on a dielectric sphere, the latter motivated by applications to sonoluminescence. In parallel to the works mentioned above, Belosludov and Nabutovskii, motivated by applications to homogeneous nucleation in superheated liquids, computed the van der Waals pressure in a spherical cavity within a homogeneous, infinite, isotropic medium [21]. The key result of Belosludov and Nabutovskii is the appearance of a size-

\footnotetext{
* zheng@uri.edu

† arvind.narayanaswamy@columbia.edu
}

dependent surface tension or surface energy at the vaporliquid interface. Though the importance of dispersion forces in phase change phenomena has long been recognized, the focus has been, to the best of our knowledge, on the effect of long-range interactions between solid and liquid on evaporation from a microlayer of liquid, or on homogeneous nucleation very close to solid surfaces [2232]. It is well known that the van der Waals/Casimir energy and stress diverges in the spherical configurations [16, 33-35]. The divergence requires the regularization of the Casimir effects by either (1) imposing a length-scale dependent cut-off in the angular momentum $[36,37]$, or (2) regularizing the expression by subtracting an "infinite" term corresponding to the bare surface tension of the liquid-vacuum interface [21, 38]. A similar regularization procedure was imposed in the case of a perfect metal shell, and the corresponding and finite result was found to be repulsive [39]. However, recent work by Kennth et al. [40] have demnstrated that such a repulsion is impossible. One of the culprits has been identified as arising from the otherwise neglected mechanical energy that is expended as a result of variations in the dielctric of the objects as they either expand or contract, requiring a more nuanced microscopic treatment of the various energy pathways in such a transformation. Regularization of the divergence of the spherical Casimir energy and stress, in this work, requires the evaluation of Casimir stress tensors on both sides of the vapor-liquid interface. They have been expressed as infinite series in terms of the spherical Riccati-Bessel (Hankel) functions, in which the "infinite" stress term in the vapor side (inside the cavity) is subtracted by the same "infinite" term in liquid (outside the cavity), that is corresponding to surface tension/interfacial energy on the boundary.

Though a vapor cavity in an infinite medium has only one interface, the corresponding problem in the planar multilayer configuration is that of a layer of vapor contained between two half-spaces of liquid, i.e., the configuration studied by Lifshitz. For a planar layer of vapor of thickness $d$, the van der Waals pressure in the vapor layer is given by $A_{h} / 6 \pi d^{3}$, where $A_{h}$ is the Hamaker coef- 


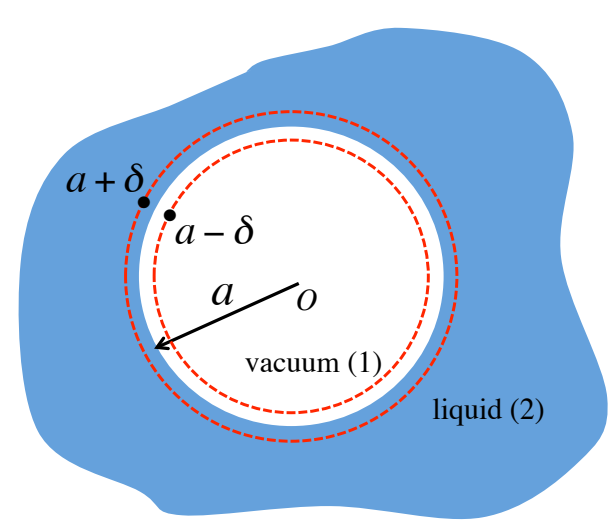

FIG. 1. Schematic of a vacuum cavity with a radius of $a$ in a liquid. Maxwell stress tensors $T_{r r, v a p}(a)$ and $T_{r r, l i q}(a)$ are evaluated at the interior and exterior of the vapor-liquid interface, corresponding to radii $r=a \mp \delta(\delta / a \rightarrow 0)$, respectively.

ficient [41, 42]. $A_{h}$ is dependent on the optical properties of the half-spaces and the vapor. At larger values of vapor layer thickness $h$, such that retardation effects are important, the pressure drops of as $h^{-4}$. The only geometric length scale in this problem is the thickness of the vapor layer, $d$. Any other length scale related to a resonant frequency in the optical properties of the media influences the pressure through $A_{h}$. Since the only geometric length scale for the cavity in an infinite medium is the radius, we should expect from dimensional analysis that the van der Waals pressure within the cavity behaves as $A_{s} / 6 \pi r^{3}$. Determining $A_{s}$, which is a spherical Hamaker coefficient, is described in Sec. II. The van der Waals pressure in the vapor layer is usually attractive (by convention $A_{h}>0$ and $A_{s}>0$ is associated with attractive pressure), i.e., the pressure of the vapor has to be larger than the pressure in the surrounding medium by $A_{h} / 6 \pi d^{3}$ (for a planar cavity) or $A_{s} / 6 \pi r^{3}$ (for a spherical cavity) to overcome the effect of attractive van der Waals forces. We are adding to the main results of Belosludov and Nabutovskii by (1) identifying a relation between the spherical and planar Hamaker coefficients, (2) giving results for liquids other than water, and (3) presenting results in a more useful form.

\section{THEORY AND CALCULATION FOR VAN DER WAALS FORCE}

To determine the Maxwell stress tensor, we rely on Rytov's theory of fluctuational electrodynamics. The crossspectral correlations of the electric and magnetic field componetnts can be written as [43, 44]:

$$
\begin{aligned}
\left\langle E_{i}(\boldsymbol{r}, \omega) E_{j}^{*}(\boldsymbol{r}, \omega)\right\rangle= & \frac{\hbar \omega^{2}}{\pi} \mu_{0} \operatorname{coth}\left(\frac{\hbar \omega}{2 k_{B} T_{l}}\right) \\
& \times \Im\left[\varepsilon(\omega) \mu(\omega) G_{e, i j}(\boldsymbol{r}, \boldsymbol{r} ; \omega)\right]
\end{aligned}
$$

$$
\begin{aligned}
\left\langle H_{i}(\boldsymbol{r}, \omega) H_{j}^{*}(\boldsymbol{r}, \omega)\right\rangle= & \frac{\hbar \omega^{2}}{\pi} \varepsilon_{0} \operatorname{coth}\left(\frac{\hbar \omega}{2 k_{B} T_{l}}\right) \\
& \times \Im\left[\varepsilon(\omega) \mu(\omega) G_{m, i j}(\boldsymbol{r}, \boldsymbol{r} ; \omega)\right]
\end{aligned}
$$

where \langle\rangle denotes the ensemble average, $\Im(z)$ denotes the imaginary part of $z, k_{B}$ is Boltzmann constant, $2 \pi \hbar$ is the Planck constant, $T_{l}$ is the absolute temperature of liquid, $\varepsilon_{0}, \mu_{0}$ are permittivity and permeability in free space respectively, and, $\varepsilon(\omega), \mu(\omega)$ are frequency dependent permittivity and permeability of the region in which $\boldsymbol{r}$ is located. $\varepsilon(\omega)=\mu(\omega)=1$ for vacuum. For liquids, $\sqrt{\varepsilon(\omega)}=n(\omega)+i \kappa(\omega)$ and $\mu(\omega)=1$. The optical data for $n$ and $\kappa$ can be obtained from Refs. [41, 45]. $G_{p, i j}(\boldsymbol{r}, \boldsymbol{r})$ is the $i j$ component of $\overline{\bar{G}}_{p}(\boldsymbol{r}, \boldsymbol{r})$, where $p=e$ refers to the electric dyadic Green's function, and $p=m$ refers to the magnetic dyadic Green's function. $\overline{\bar{G}}_{e}(\boldsymbol{r}, \boldsymbol{r})$ and $\overline{\bar{G}}_{m}(\boldsymbol{r}, \boldsymbol{r})$ are electromagnetic duals of each other [43? ]. The van der Waals pressure on the interior and exterior surfaces of a cavity in an otherwise homogeneous medium (see Fig. 1) can be obtained from the $\hat{r} \hat{r}$ component of the electromagnetic or Maxwell stress tensor. The $r r$ component of the Maxwell stress tensor can be expressed in terms of $G_{e}(\boldsymbol{r}, \boldsymbol{r})$ and $G_{m}(\boldsymbol{r}, \boldsymbol{r})$ as [44]:

$$
\begin{aligned}
\mathcal{T}_{r r}(r)= & \int_{0}^{\infty} d \omega \frac{\hbar \omega^{2}}{\pi c^{2}} \operatorname{coth}\left(\frac{\hbar \omega}{2 k_{B} T}\right) \\
& \times \Im\left\{\varepsilon(\omega) \mu(\omega)\left[S_{e}(\boldsymbol{r}, \omega)+S_{m}(\boldsymbol{r}, \omega)\right]\right\}
\end{aligned}
$$

where $S_{e}(\boldsymbol{r}, \omega)=G_{e, \hat{r} \hat{r}}(\boldsymbol{r}, \boldsymbol{r} ; \omega)-\frac{1}{2} \operatorname{Tr} \overline{\bar{G}}_{e}(\boldsymbol{r}, \boldsymbol{r} ; \omega), S_{m}=$ $G_{m, \hat{r} \hat{r}}(\boldsymbol{r}, \boldsymbol{r} ; \omega)-\frac{1}{2} \operatorname{Tr} \overline{\bar{G}}_{m}(\boldsymbol{r}, \boldsymbol{r} ; \omega) . \operatorname{Tr}$ is the trace of the tensor, that is $\operatorname{Tr} \overline{\bar{G}}_{p}=G_{p, \hat{r} \hat{r}}+G_{p, \hat{\theta} \hat{\theta}}+G_{p, \hat{\phi} \hat{\phi}}$. The integral on the right hand side of Eq. 2 is of the form $\int_{0^{+}}^{\infty} d \omega \operatorname{coth}\left(\frac{\hbar \omega}{2 k_{B} T_{l}}\right) \Im f(\omega)$, in which the function $f(\omega+i \xi)$ is analytic in the upper-half of the complex plane $(\xi>0)$. Since $G(\boldsymbol{r}, \omega)$ is analytic in the upper-half of the complex plane, the integral along the real frequency axis can be transformed into summation over Matsubara frequencies, $i \xi_{n}=i n 2 \pi k_{B} T_{l} / \hbar$, as $-\frac{2 \pi k_{B} T}{\hbar} \sum_{n=0}^{\infty}{ }^{\prime} f\left(i \xi_{n}\right) . \quad \sum_{n=0}^{\infty}$, indicates that the $n=0$ term is multiplied by $1 / 2[1]$.

$S_{p}(\boldsymbol{r}, \omega)$ can be split into two parts: (1) a part which arises from the dyadic Green's function in an infinite homogeneous medium, and (2) a part which arises from the scattered dyadic Green's function due to the presence of boundaries, as $S_{p}(\boldsymbol{r}, \omega)=S^{(o)}(\boldsymbol{r}, \omega)+S_{p}^{(s c)}(\boldsymbol{r}, \omega)$. Since $S^{(o)}(\boldsymbol{r}, \omega)$ is the stress tensor contribution from the homogeneous medium, there is no distinction between the $p=e$ or $p=m$ contributions. The dyadic Green's function $\overline{\bar{G}}^{(o)}(\boldsymbol{r}, \tilde{\boldsymbol{r}} ; \omega)$ that gives rise to $S^{(o)}(\boldsymbol{r}, \omega)$ is given by:

$$
\overline{\bar{G}}^{(o)}(\boldsymbol{r}, \tilde{\boldsymbol{r}} ; \omega)=i k_{i} \sum_{\substack{m=-l, l=0 \\
l=l}}^{\substack{l=\infty \\
l=0}}\left[\begin{array}{l}
\boldsymbol{M}_{l, m}^{(3)}\left(k_{i} \boldsymbol{r}\right) \boldsymbol{M}_{l,-m}^{(1)}\left(k_{i} \tilde{\boldsymbol{r}}\right) \\
+\boldsymbol{N}_{l, m}^{(3)}\left(k_{i} \boldsymbol{r}\right) \boldsymbol{N}_{l,-m}^{(1)}\left(k_{i} \tilde{\boldsymbol{r}}\right)
\end{array}\right](3)
$$


when $|\boldsymbol{r}|>|\tilde{\boldsymbol{r}}| . k_{i}=\omega \sqrt{\varepsilon_{i}(\omega) \mu_{i}(\omega)} / c$ is the wavenumber in region $i(i=1,2$ refer to the cavity and medium respectively), $\boldsymbol{M}_{l, m}^{(p)}\left(k_{i} a\right)$ and $\boldsymbol{N}_{l, m}^{(p)}\left(k_{i} a\right)$ are vector spherical wave functions of order $(l, m)$, and superscript $(p)$ refers to the radial behavior of the waves, given by [46]

$$
\begin{gathered}
\boldsymbol{M}_{l, m}^{(p)}\left(k_{i} a\right)=\frac{u_{l}^{(p)}\left(k_{i} a\right)}{k_{i} a \sqrt{l(l+1)}}\left(\hat{\theta} \frac{i m Y_{l, m}}{\sin \theta}-\hat{\phi} \frac{\partial Y_{l, m}}{\partial \theta}\right) \\
\boldsymbol{N}_{l, m}^{(p)}\left(k_{i} a\right)=\hat{r} \frac{u_{l}^{(p)}\left(k_{i} a\right)}{\left(k_{i} a\right)^{2}} \sqrt{l(l+1)} Y_{l, m} \\
+\frac{v_{l}^{(p)}\left(k_{i} a\right)}{k_{i} a \sqrt{l(l+1)}}\left(\hat{\theta} \frac{\partial Y_{l, m}}{\partial \theta}+\hat{\phi} \frac{i m Y_{l, m}}{\sin \theta}\right)
\end{gathered}
$$

For $p=1$, both $\boldsymbol{M}$ and $\boldsymbol{N}$ waves are regular vector spherical waves and $u_{l}^{(1)}$ is the spherical Riccati-Bessel function of first kind of order $l$. For $p=3$, both $M$ and $\boldsymbol{N}$ waves are outgoing spherical waves and $u_{l}^{(3)}$ is the spherical Riccati-Hankel function of first kind of order $l$. $v_{l}^{(p)}$ is first derivative of Riccati-Bessel (Hankel) function, defined as $v_{l}^{(p)}(x)=\frac{d}{d x}\left[u_{l}^{(p)}(x)\right] . Y_{l, m}$ is the spherical harmonic of order $(l, m)$. Substituting Eqs. 3, 4a and $4 \mathrm{~b}$ into Eq. 2, we obtain the homogeneous contribution to stress tensor as:

$$
\begin{aligned}
\mathcal{T}_{r r}^{(o)}(a)= & \Re \int_{0}^{\infty} d \omega \frac{\hbar \omega^{3}}{4 \pi^{2} c^{3}} \operatorname{coth}\left[\frac{\hbar \omega}{2 k_{B} T_{l}}\right] \sum_{l=0}^{l=\infty}(2 l+1) \\
\times & {\left[\begin{array}{l}
\left(1-\frac{l(l+1)}{k_{i}^{2} a^{2}}\right) \frac{u_{l}^{(3)}\left(k_{i} a\right)}{k_{i} a} \frac{u_{l}^{(1)}\left(k_{i} a\right)}{k_{i} a} \\
+\frac{v_{l}^{(3)}\left(k_{i} a\right)}{k_{i} a} \frac{v_{l}^{(1)}\left(k_{i} a\right)}{k_{i} a}
\end{array}\right] }
\end{aligned}
$$

Applying the following summation identities of spherial Riccati-Bessel (Hankel) function

$$
\begin{gathered}
\sum_{l=0}^{l=\infty}(2 l+1)\left(\frac{u_{l}^{(1)}(x)}{x}\right)^{2}=1, \\
\sum_{l=0}^{l=\infty} l(l+1)(2 l+1)\left(\frac{u_{l}^{(1)}(x)}{x}\right)^{2}=0, \\
\sum_{l=0}^{l=\infty}(2 l+1)\left(\frac{v_{l}^{(1)}(x)}{x}\right)^{2}=\frac{1}{3},
\end{gathered}
$$

we can see that the free space or homogeneous dyadic Green's function does not contribute to a configurationdependent (radius of vacuum region for the configuration in Fig. 1) stress tensor.

Because the homogeneous part of the dyadic Green's function does not contribute to van der Waals stress tensor, we focus only on the scattered part of dyadic Green's functions from now on. The expressions for $\overline{\bar{G}}_{e}^{(s c)}(\boldsymbol{r}, \tilde{\boldsymbol{r}} ; \omega)$ when $\boldsymbol{r}, \tilde{\boldsymbol{r}} \in V_{1}$ and $\boldsymbol{r}, \tilde{\boldsymbol{r}} \in V_{2}$ are given by:

$$
\begin{aligned}
& \overline{\bar{G}}_{e}^{(s c)}(\boldsymbol{r}, \tilde{\boldsymbol{r}} ; \omega)=i k_{1} \times \\
& \sum_{\substack{m=-l, l=0}}^{\substack{l=\infty, m=l}}(-1)^{m}\left[\begin{array}{l}
R_{1, l}^{M} \boldsymbol{M}_{l, m}^{(1)}\left(k_{1} \boldsymbol{r}\right) \boldsymbol{M}_{l,-m}^{(1)}\left(k_{1} \tilde{\boldsymbol{r}}\right)+ \\
R_{1, l}^{N} \boldsymbol{N}_{l, m}^{(1)}\left(k_{1} \boldsymbol{r}\right) \boldsymbol{N}_{l,-m}^{(1)}\left(k_{1} \tilde{\boldsymbol{r}}\right)
\end{array}\right] \\
& \overline{\bar{G}}_{e}^{(s c)}(\boldsymbol{r}, \tilde{\boldsymbol{r}} ; \omega)=i k_{2} \times \\
& \sum_{\substack{m=-\infty, l=0}}^{m=l}(-1)^{m}\left[\begin{array}{l}
R_{2, l}^{M} \boldsymbol{M}_{l, m}^{(3)}\left(k_{2} \boldsymbol{r}\right) \boldsymbol{M}_{l,-m}^{(3)}\left(k_{2} \tilde{\boldsymbol{r}}\right)+ \\
R_{2, l}^{N} \boldsymbol{N}_{l, m}^{(3)}\left(k_{2} \boldsymbol{r}\right) \boldsymbol{N}_{l,-m}^{(3)}\left(k_{2} \tilde{\boldsymbol{r}}\right)
\end{array}\right]
\end{aligned}
$$

where, $R_{i, l}^{j}$ is the Mie refection coefficient due to the source in region $i$ due to $j(=\boldsymbol{M}, \boldsymbol{N})$ waves. The Mie reflection coefficients $R_{1, l}^{M}$ and $R_{2, l}^{M}$ due to $M$ waves are obtained from the boundary condition equations as [47]:

$$
\begin{gathered}
R_{1, l}^{M}=-\frac{\left(\frac{k_{1}}{\mu_{1}} \frac{v_{l}^{(3)}\left(k_{1} a\right)}{u_{l}^{(3)}\left(k_{1} a\right)}-\frac{k_{2}}{\mu_{2}} \frac{v_{l}^{(3)}\left(k_{2} a\right)}{u_{l}^{(3)}\left(k_{2} a\right)}\right)}{\left(\frac{k_{1}}{\mu_{1}} \frac{v_{l}^{(1)}\left(k_{1} a\right)}{u_{l}^{(1)}\left(k_{1} a\right)}-\frac{k_{2}}{\mu_{2}} \frac{v_{l}^{(3)}\left(k_{2} a\right)}{u_{l}^{(3)}\left(k_{2} a\right)}\right) u_{l}^{(3)}\left(k_{1} a\right)} \\
u_{l}^{(1)}\left(k_{1} a\right) \\
R_{2, l}^{M}=-\frac{\left(\frac{k_{2}}{\mu_{2}} \frac{v_{l}^{(1)}\left(k_{2} a\right)}{u_{l}^{(1)}\left(k_{2} a\right)}-\frac{k_{1}}{\mu_{1}} \frac{v_{l}^{(1)}\left(k_{1} a\right)}{u_{l}^{(1)}\left(k_{1} a\right)}\right)}{\left(\frac{k_{2}}{\mu_{2}} \frac{v_{l}^{(3)}\left(k_{2} a\right)}{u_{l}^{(3)}\left(k_{2} a\right)}-\frac{k_{1}}{\mu_{1}} \frac{v_{l}^{(1)}\left(k_{1} a\right)}{u_{l}^{(1)}\left(k_{1} a\right)}\right)} \frac{u_{l}^{(1)}\left(k_{2} a\right)}{u_{l}^{(3)}\left(k_{2} a\right)}
\end{gathered}
$$

Similarly, $R_{1, l}^{N}$ and $R_{2, l}^{N}$ due to $N$ waves can be obtained by simply exchanging functions of $u_{l}$ and $v_{l}$. $\overline{\bar{G}}_{m}^{(s c)}(\boldsymbol{r}, \tilde{\boldsymbol{r}} ; \omega)$ can be obtained by replacing $\varepsilon_{i}$ by $\mu_{i}$ and vice-versa $(i=1,2)$ in Eq. $7 \mathrm{a}-9$ [43? ]. Substituting the scattered part of spherical dyadic Green's functions Eqs. 7a and 7b into Eq. 2, we find that the $\hat{r} \hat{r}$ component of the stress tensor on either side of the vapor-liquid interface, i.e., at $r=a \pm \delta$ as $\delta \rightarrow 0$, can be written as:

$$
\begin{aligned}
\mathcal{T}_{r r, v a p}^{(s c)}(a)=-\Re \int_{0}^{\infty} & d \omega \frac{\hbar \omega^{2}}{4 \pi^{2} c^{2}} \frac{1}{k_{1} a^{2}} \operatorname{coth}\left(\frac{\hbar \omega}{2 k_{B} T_{l}}\right) \sum_{l=0}^{\infty}(2 l+1)\left(R_{1, l}^{(M)}+R_{1, l}^{(N)}\right) \\
\times & {\left[\left(1-\frac{l(l+1)}{k_{1}^{2} a^{2}}\right) u_{l}^{(1) 2}\left(k_{1} a\right)+v_{l}^{(1) 2}\left(k_{1} a\right)\right] }
\end{aligned}
$$




$$
\begin{aligned}
\mathcal{T}_{r r, l i q}^{(s c)}(a)=-\Re \int_{0}^{\infty} & d \omega \frac{\hbar \omega^{2}}{4 \pi^{2} c^{2}} \frac{\varepsilon_{2} \mu_{2}}{k_{2} a^{2}} \operatorname{coth}\left(\frac{\hbar \omega}{2 k_{B} T_{l}}\right) \sum_{l=0}^{l=\infty}(2 l+1)\left(R_{2, l}^{(M)}+R_{2, l}^{(N)}\right) \\
\times & {\left[\left(1-\frac{l(l+1)}{k_{2}^{2} a^{2}}\right) u_{l}^{(3) 2}\left(k_{2} a\right)+v_{l}^{(3) 2}\left(k_{2} a\right)\right] }
\end{aligned}
$$

where the subscripts vap and liq refer to the vapor and liquid regions, namely, inside and outside the cavity. It can be shown that the stress tensor in radial direction on the interior side of the vapor-liquid interface (Eq. 10a) is divergent when summing up the infinite series of RiccatiBessel (Hankel) functions with respect to $l$. It is also well known that the van der Waals/Casimir energy and stress diverges in the spherical configurations [16, 33-35], that requires a proper regularization of the Casimir effects. Here, the treatment of interfacial forces in a bounded geometry is taken into account. It yields the corresponding mechanical stress/energy on the exterior side of the vapor-liquid interface, and its "infinite" stress term in liquid (Eq. 10b) subtracts the same "infinite" term in vapor (Eq. 10a), and it gives rise to the convergent and finite result (please refer to Eq. 11 in Sec. III).

\section{RESULTS AND DISCUSSION}

In the preceding section, we have derived the expressions for the van der Waals pressure of a vacuum cavity of radius $a$ surrounded by a dissipative liquid medium in Eqs. 10a and 10b. The van der Waals pressure in a vacuum layer sandwiched between two half-spaces is given by the $z z$ component of the Maxwell stress tensor, $\mathcal{T}_{z z}^{(s c)}(d)$, where the $z$-axis is perpendicular to the planar interfaces. $\mathcal{T}_{z z}^{(s c)}(d)$ is related to the optical properties of the materials involved and the thickness of the vacuum layer, $d$, as $\mathcal{T}_{z z}^{(s c)}(d)=A_{h} / 6 \pi d^{3}[41,42]$. The thickness of vacuum film $d$ is the unique dimensional parameter for a thin-film configuration. Similarly, in this work, the radius $a$ of vacuum cavity embedded in dissipative medium is the unique geometric parameter. Hence, we are able to define a spherical Hamaker function with a definition similar to the planar Hamaker function, i.e., $\mathcal{T}_{r r, v a p}^{(s c)}(a)=A_{s} / 6 \pi a^{3}$, which can be used to evaluate the van der Waals pressure on the interior surface of a spherical cavity of radius $a$. In Table I, we list the planar and spherical Hamaker coefficients, $A_{h}$ and $A_{s}$, as well as $A_{s} / A_{h}$ for various materials. The optical properties needed to calculate $A_{h}$ and $A_{s}$ are obtained from Refs. $[41,45]$. It can be seen clearly that the dimensionless ratio of Hamaker function $A_{s} / A_{h}$ is approximately around $9.0 \sim 9.5$. The increase in spherical Hamaker constant is attributed to a stronger confinement of fluctuational electromagnetic waves within a nanoscale sphere (a 3-D cavity) in comparison to a thin film (a planar cavity).

Let the hydrostatic pressures in the vapor and liquid regions be $p_{v a p}$ and $p_{l i q}$ respectively. The total $\hat{r} \hat{r}$
TABLE I. Planar and spherical Hamaker constants for various materials. Planar Hamaker constant $A_{h}$ has been calculated by using Lifshitz' technique, in comparison with the values as shown in Hough and White's paper [41]. $A_{s}$, the spherical Hamaker constant for a vacuum cavity in a homogeneous

\begin{tabular}{|c|c|c|}
\hline \multirow[t]{2}{*}{ Substance } & $A_{h}(\mathrm{zJ})$ & $A_{s}(\mathrm{zJ})$ \\
\hline & Ours Hough [41] & \\
\hline Water & 54.2155 .1 & 519.19 \\
\hline n-Pentane & 37.5137 .5 & 357.72 \\
\hline n-Hexane & 40.6840 .7 & 378.60 \\
\hline n-Heptane & 43.1143 .2 & 396.38 \\
\hline n-Octane & $44.94 \quad 45.0$ & 405.28 \\
\hline n-Nonane & 46.6246 .6 & 443.84 \\
\hline n-Decane & 48.1948 .2 & 454.21 \\
\hline Polystyrene & 65.8765 .8 & 606.03 \\
\hline Polyvinylchloride & 78.1377 .8 & 697.58 \\
\hline Polyisoprene & $60.06 \quad 59.9$ & 561.53 \\
\hline
\end{tabular}
substance, can be calculated using Eq. 12 .

stress tensor on either side of the interface in the vapor and liquid regions are given by $-p_{\text {vap }}+\mathcal{T}_{r r, v a p}^{(s c)}(a)$ and $-p_{l i q}+\mathcal{T}_{r r, l i q}^{(s c)}(a)$ respectively. Balance of forces at the interface yields a modified Young-Laplace equation as shown below:

$$
\left(p_{\text {vap }}-\mathcal{T}_{\text {rr }, \text { vap }}^{(s c)}(a)\right)-\left(p_{\text {liq }}-\mathcal{T}_{r r, l i q}^{(s c)}(a)\right)=\frac{2 \sigma_{\infty}\left(T_{l}\right)}{a}
$$

where $\sigma_{\infty}\left(T_{l}\right)$ is temperature dependent surface tension. The stress tensors $\mathcal{T}_{r r, v a p}^{(s c)}(a)$ and $\mathcal{T}_{r r, l i q}^{(s c)}(a)$ are plotted for water and n-Heptane (we chose n-Heptane as an example of an hydrocarbon/non-polar material) in Fig. 2. It can be seen clearly that the $\hat{r} \hat{r}$ component $\mathcal{T}_{r r, v a p}^{(s c)}(a)$ obeys a $a^{-3}$ rule, while $\mathcal{T}_{r r, l i q}^{(s c)}(a)$ behaves as $a^{-1}$, which is negligible compared to the inner stress unless a cavity grows up to one of radius as large as $40 \mathrm{~nm}$. Since $a \mathcal{T}_{r r, \text { liq }}^{(s c)}(a) \ll \sigma_{\infty}, \mathcal{T}_{r r, l i q}^{(s c)}(a)$ does not contribute significantly to $\sigma_{\infty} ; a \mathcal{T}_{r r, l i q}^{(s c)}(a) / \sigma_{\infty} \approx 10^{-4}$ for water and $10^{-3}$ for n-Heptane.

Manipulation of Eq. 11 gives rise to a temperature as well as size dependent surface energy $\sigma\left(a, T_{l}\right)$ of a cavity of radius $a$ at temperature $T_{l}$ (neglecting the term $\mathcal{T}_{r r, l i q}^{(s c)}(a)$ as it is negligible compared to $\mathcal{T}_{r r, v a p}^{(s c)}(a)$ for small radii):

$$
\sigma\left(a, T_{l}\right) \approx \sigma_{\infty}\left(T_{l}\right)+\frac{a}{2} \mathcal{T}_{r r, v a p}^{(s c)}(a)=\sigma_{\infty}\left(T_{l}\right)+\frac{A_{s}}{12 \pi a^{2}}
$$

It is known that surface tension $\sigma_{\infty}$ of liquid varies as the liquid temperature $T_{l}$, and it also depends on the critical temperature $T_{c}$ and critical pressure $P_{c}$ of the liquid. 


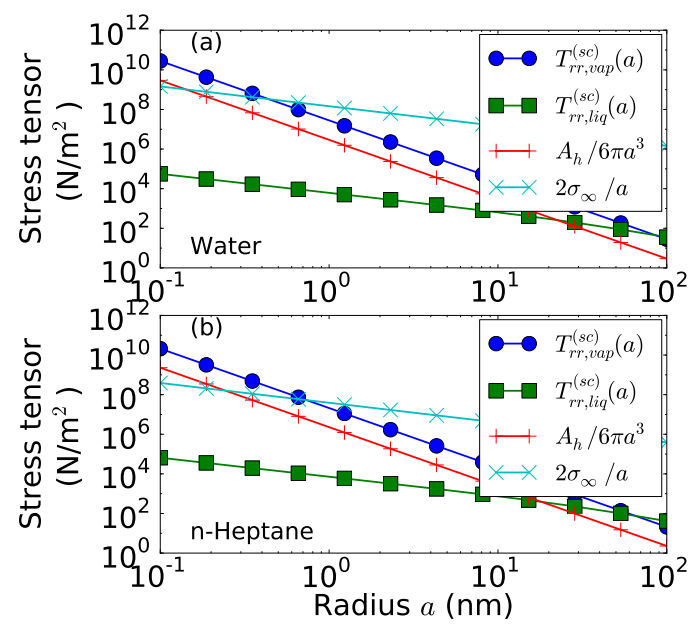

FIG. 2. (a) Stress tensor at surface of a vacuum cavity in water. The blue curve with circular markers and the green curve with square markers are Maxwell stress tensors at the interior $\left[\mathcal{T}_{r r, v a p}^{(s c)}(a) \sim a^{-3}\right]$ and exterior $\left[\mathcal{T}_{r r, l i q}^{(s c)}(a) \sim a^{-1}\right]$ of the vacuum-water interface. The van der Waals pressure in a vacuum layer between two half-spaces of water (light blue curve with "+" markers) and the capillary pressure due to surface tension of water at room temperature $T_{l}=300 \mathrm{~K}$ (cyan curve with " $\times$ " markers) are also shown. (b) Similar plot for n-Heptane.

We use Vargaftik's expression [48] for surface tension for water, which is given by:

$$
\sigma_{\infty}\left(T_{l}\right)=B\left(1-\frac{T_{l}}{T_{c}}\right)^{\mu}\left[1+b\left(1-\frac{T_{l}}{T_{c}}\right)\right],
$$

where $B=235.8 \times 10^{-3} \mathrm{~N} / \mathrm{m}, b=-0.625$ and $\mu=1.256$. Similarly, we use the expression proposed by Riedel [49] and Carey [50] for surface tension for hydrocarbons, which is given by:

$$
\sigma_{\infty}\left(T_{l}\right)=P_{c}^{2 / 3} T_{c}^{1 / 3}\left(\frac{0.133 R_{c}-0.281}{1000}\right)\left(1-\frac{T_{l}}{T_{c}}\right)^{11 / 9}
$$

$R_{c}$ is the Riedel parameter $[49,50]$ defined as

$$
R_{c}=0.9076\left(1+\frac{T_{b}}{T_{c}} \ln P_{c}\right)\left(1-\frac{T_{b}}{T_{c}}\right)^{-1}
$$

where $T_{b}$ is the boiling point of the hydrocarbon. In these relations, temperature $T_{b}$ and $T_{c}$ are in Kelvin, $P_{c}$ is in atmospheres. The critical temperature and pressure, boiling point, and Riedel parameter at standard atmospheric pressure for n-Heptane are $540.3 \mathrm{~K}, 26.9 \mathrm{~atm}$, $371.6 \mathrm{~K}$, and 9.49 respectively.

In Fig. 2, along with the Maxwell stress tensor components $\mathcal{T}_{\text {rr,vap }}^{(s c)}(a)$ and $\mathcal{T}_{r r, \text { liq }}^{(s c)}(a)$, we also show the variation with radius $a$ of the excess pressure within the vapor cavity due to surface tension, $2 \sigma_{\infty} / a$, and the van der Waals pressure in a film of thickness $a, A_{h} / 6 \pi a^{3}$. Equation 12 suggests that we can define a transition radius $r_{t r}$ when

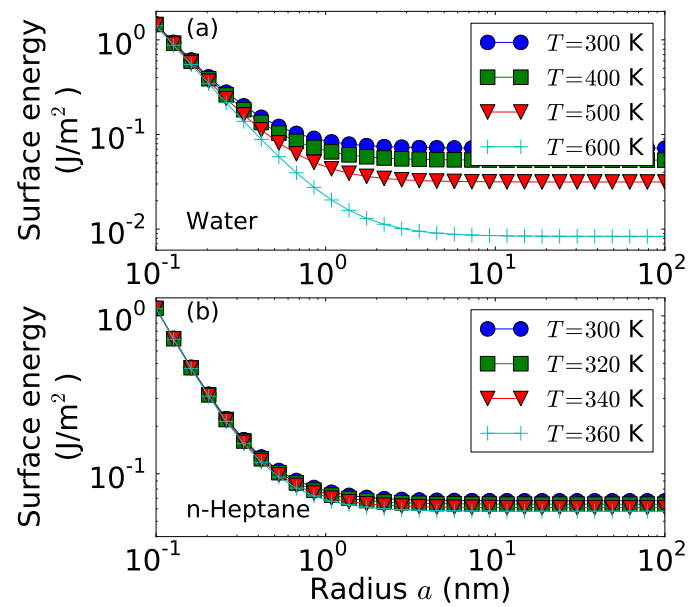

FIG. 3. Surface energy $\sigma\left(a, T_{l}\right)=\sigma_{\infty}\left(T_{l}\right)+A_{s} / 12 \pi a^{2}$ varies as radius $a$ at different temperature, for (a) water and (b) n-Heptane.

the curvature dependent contribution to pressure within the vapor cavity becomes comparable to the usual surface tension dependent part. Here, we define $r_{t r}$ to be such that $\mathcal{T}_{r r, v a p}^{(s c)}(a)$ is $10 \%$ of $2 \sigma_{\infty} / a$. Such a radius is given by:

$$
\frac{A_{s}}{6 \pi r_{t r}^{3}}=0.1 \frac{2 \sigma_{\infty}\left(T_{l}\right)}{r_{t r}} \Rightarrow r_{t r}\left(T_{l}\right)=\sqrt{\frac{A_{s}}{1.2 \pi \sigma_{\infty}\left(T_{l}\right)}}
$$

As $a \gg r_{t r}$, surface tension dominates, whereas $a<r_{t r}$, van der Waals pressure plays a significant role in surface energy of a nanoscale cavity, as shown in Fig. 3. Figures $3 \mathrm{a}$ and $3 \mathrm{~b}$ show the dependence of surface energy, $\sigma\left(a, T_{l}\right)$ [Eq. 12] on temperature and cavity size for water and nHeptane respectively. Substituting Eqs. 13 and 14 and spherical Hamaker constants $\left(A_{s}\right)$ from Table I into Eq. 16 , it can be found that the transition radius is $r_{t r} \approx 10$ $\mathrm{nm}$ and $\approx 40 \mathrm{~nm}$ for water and $\mathrm{n}$-Heptane respectively. Surface energy $\sigma\left(a, T_{l}\right) \approx A_{s} / 12 \pi a^{2}$ when $a \ll r_{t r}$, and $\sigma\left(a, T_{l}\right) \approx \sigma_{\infty}\left(T_{l}\right)$ when $a \gg r_{t r}$.

We apply this result to the theory of homogeneous nucleation of bubbles in liquids. A key quantity in the theory of homogeneous nucleation is the equilibrium radius, $r_{e}\left(T_{l}, P_{l}\right)$, which is the radius at which vapor bubbles are in equilibrium (unstable) with a liquid at temperature $T_{l}$ and pressure $P_{l}$. The dependence of $r_{e}$ on $T_{l}$ and $P_{l}$ is given by [50]:

$$
r_{e}\left(T_{l}, P_{l}\right)=\frac{2 \sigma_{\infty}\left(T_{l}\right)}{P_{s a t}\left(T_{l}\right) \exp \left(\frac{v_{l}\left[P_{l}-P_{s a t}\left(T_{l}\right)\right]}{R T_{l}}\right)-P_{l}}
$$

where $T_{l}<T_{c}$ and $P_{l}<P_{\text {sat }}\left(T_{l}\right)$ for a superheated liquid. Figure 4 shows the dependence of equilibrium radius of a cavity on temperature $\left(T_{l}<0.95 T_{c}\right)$ and pressure $P_{l}=P_{a t m}=1.01 \times 10^{5} \mathrm{~Pa}$ and $P_{l}=10 P_{a t m}$ for water and n-Heptane. It can be seen that the equilibrium 
radius decreases with increasing temperature and, eventually, becomes comparable to the transition radius. To illustrate this effect better, we plot the ratio of radii of $r_{t r}\left(T_{l}\right)$ and $r_{e}\left(T_{l}, P_{l}\right)$ as a function of $T_{l}$ at different liquid pressures in Fig. 5. When $r_{t r}\left(T_{l}\right) / r_{e}\left(T_{l}, P_{l}\right) \gtrsim 1$, then classical theory of homogeneous nucleation should be modified in order to take curvature dependent van der Waals contribution into effect. The numerical val-

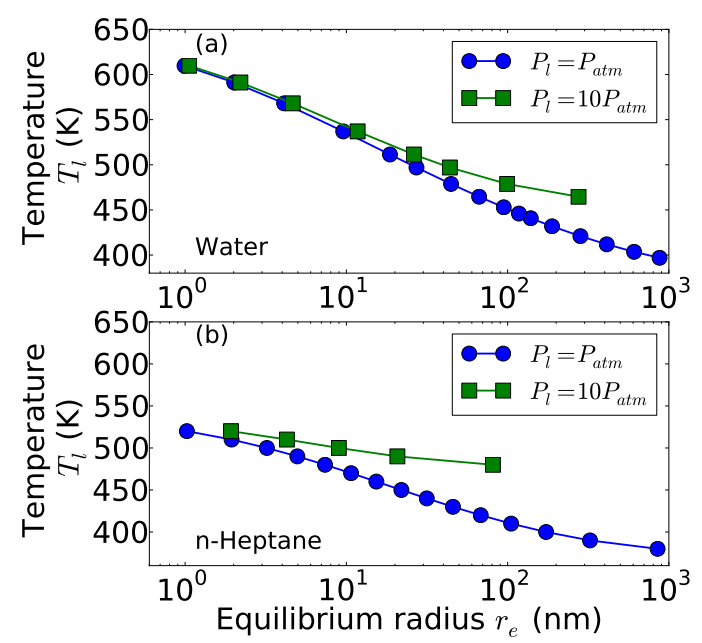

FIG. 4. Temperature and pressure dependent critical size, $r_{e}\left(T_{l}, P_{l}\right)$, of a cavity surrounded by a superheated liquid, for (a) water and (b) n-Heptane.

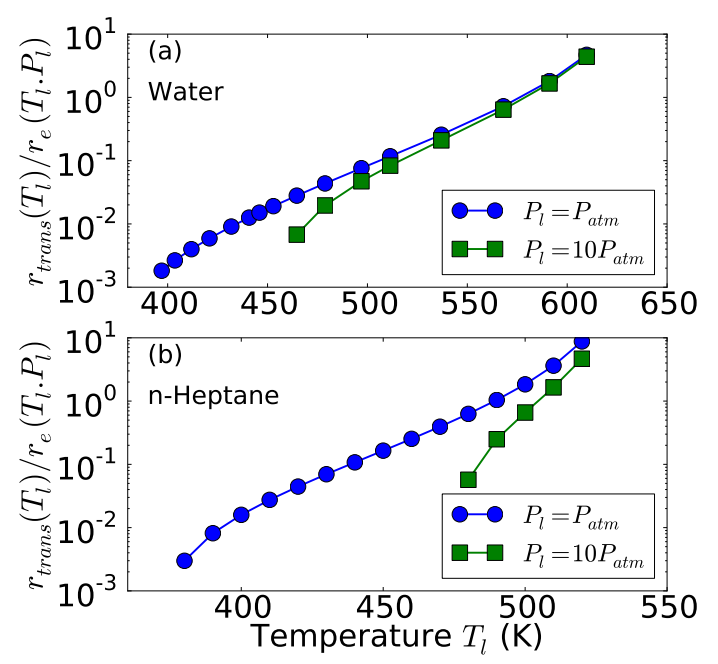

FIG. 5. Ratio of the transition radius, $r_{t r}\left(T_{l}\right)$, and the equilibrium radius, $r_{e}\left(T_{l} . P_{l}\right)$, for (a) water and (b) n-Heptane. ues of $A_{s}$ given here cannot be extended to the case when $T_{l} \gtrsim 0.95 T_{c}$ because the vapor inside the cavity can no longer be approximated as vacuum. Making appropriate allowance for this by defining a density-dependent dielectric function of the vapor phase can extend this theoretical model up to $T_{l}=T_{c}$. However, the anaylsis of van der Waals interactions at all temperatures up to $T_{c}$, and the effect of van der Waals interactions on homogeneous nucleation will be pursued in a separate manuscript.

\section{SUMMARY}

We have used fluctuational electrodynamics to determine the van der Waals pressure inside a spherical nanocavity within a homogeneous, isotropic, infinite medium. The van der Waals pressure inside the cavity of radius $a$ is shown to behave as $A_{s} / 6 \pi a^{3}$, in agreement with dimensional analysis. The size-dependent surface energy (or tension) can be written as $\sigma\left(a, T_{l}\right)=$ $\sigma_{\infty}\left(T_{l}\right)+A_{s} / 12 \pi a^{2}$. Unlike $\sigma_{\infty}, A_{s}$ only has a weak temperature dependence when $T_{l}<0.95 T_{c}$. We define a transition radius at which the van der Waals pressure contributes an unnegligible amount (assumed to be 10\% here) to the surface tension of liquid, and compare it with the prediction of the classical theory of homogeneous nucleation for the equilibrium radius of a vapor cavity surrounded by a superheated liquid. Since the surface energy of the liquid-vapor interface is important to the classical theory of homogeneous nucleation, we expect the work presented here to have a direct impact on our understanding of homogeneous nucleation inside micro/nanocavity.

\section{ACKNOWLEDGMENTS}

This work is funded by the Start-up Grant through the College of Engineering at the University of Rhode Island.

\section{REFERENCES}

[1] E. Lifshitz, Sov. Phys. JETP 2, 73 (1956).

[2] S. Rytov, Theory of Electric Fluctuations and Thermal Radiation (Air Force Cambridge Research Center, Bedford, Mass., 1959), Tech. Rep. (AFCRC-TR-59-162,
1967).

[3] I. E. Dzyaloshinskii, E. Lifshitz, and L. P. Pitaevskii, Physics-Uspekhi 4, 153 (1961). 
[4] V. A. Parsegian and B. W. Ninham, Journal of Theoretical Biology 38, 101 (1973).

[5] Y. Zheng and A. Narayanaswamy, Physical Review A 83, 042504 (2011).

[6] A. Narayanaswamy and Y. Zheng, Physical Review A 88, 012502 (2013).

[7] M. Antezza, L. P. Pitaevskii, S. Stringari, and V. B. Svetovoy, Physical Review A 77, 022901 (2008).

[8] Y. Zheng and A. Narayanaswamy, Physical Review A 89, 022512 (2014).

[9] Y. Zheng, Advances in Condensed Matter Physics 2015 (2015).

[10] A. W. Rodriguez, F. Capasso, and S. G. Johnson, Nature photonics 5, 211 (2011).

[11] S. Kondrat, L. Harnau, and S. Dietrich, The Journal of chemical physics 131, 204902 (2009).

[12] E. Noruzifar and M. Oettel, Physical Review E 79, 051401 (2009).

[13] H. Gies and K. Klingmüller, Physical review letters 96, 220401 (2006).

[14] M. Bordag, Physical Review D 73, 125018 (2006).

[15] B. Geyer, G. Klimchitskaya, and V. Mostepanenko, The European Physical Journal C-Particles and Fields 57, 823 (2008).

[16] T. H. Boyer, Physical Review 174, 1764 (1968).

[17] K. A. Milton, L. L. DeRaad Jr, and J. Schwinger, Annals of Physics 115, 388 (1978).

[18] J. Schwinger, J. L. L. DeRaad, and K. A. Milton, Ann. Phys. (N. Y.) 115, 1 (1978).

[19] K. A. Milton, Annals of Physics 127, 49 (1980).

[20] K. A. Milton and Y. J. Ng, Physical Review E 55, 4207 (1997).

[21] V. Belosludov and V. Nabutovskiy, Zh. eksp. i teor. fiz 68, 2177 (1975).

[22] V. P. Carey and A. P. Wemhoff, International journal of heat and mass transfer 48, 5431 (2005).

[23] A. P. Wemhoff and V. P. Carey, Microscale Thermophysical Engineering 9, 331 (2005).

[24] S. J. Gokhale, J. L. Plawsky, and P. C. Wayner Jr, Journal of colloid and interface science 259, 354 (2003).

[25] P. C. Wayner Jr, International Journal of Heat and Mass Transfer 25, 707 (1982).

[26] P. Wayner Jr, AIChE journal 45, 2055 (1999).

[27] S. DasGupta, I. Y. Kim, and P. C. Wayner, Journal of Heat Transfer 116, 1007 (1994).
[28] A. Chatterjee, J. L. Plawsky, and P. C. Wayner Jr, Advances in colloid and interface science 168, 40 (2011).

[29] J. N. Chung, T. Chen, and S. Maroo, Frontiers in Heat and Mass Transfer (FHMT) 2 (2011).

[30] S. C. Maroo and J. N. Chung, International Journal of Heat and Mass Transfer 53, 3335 (2010).

[31] S. C. Maroo and J. N. Chung, Journal of Heat Transfer 135, 061501 (2013).

[32] J. L. Plawsky, A. G. Fedorov, S. V. Garimella, H. B. Ma, S. C. Maroo, L. Chen, and Y. Nam, Nanoscale and Microscale Thermophysical Engineering 18, 251 (2014).

[33] V. Nesterenko and I. Pirozhenko, Physical Review D 57, 1284 (1998).

[34] G. Barton, Journal of Physics A: Mathematical and General 34, 4083 (2001).

[35] S. Lim and L. Teo, New Journal of Physics 11, 013055 (2009).

[36] I. Affleck, arXiv preprint cond-mat/9512099 (1995).

[37] X. Ai-Min and C. Xiao-Song, Communications in Theoretical Physics 50, 1317 (2008).

[38] V. M. Nabutovskii and V. R. Belosludov, International Journal of Modern Physics B 3, 171 (1989).

[39] O. Kenneth, I. Klich, A. Mann, and M. Revzen, Physical review letters 89, 033001 (2002).

[40] O. Kenneth and I. Klich, Physical review letters 97, 160401 (2006).

[41] D. B. Hough and L. R. White, Advances in Colloid and Interface Science 14, 3 (1980).

[42] J. N. Israelachvili, Intermolecular and surface forces: revised third edition (Academic press, 2011).

[43] A. Narayanaswamy and G. Chen, Journal of Quantitative Spectroscopy and Radiative Transfer 111, 1877 (2010).

[44] A. Narayanaswamy and Y. Zheng, Journal of Quantitative Spectroscopy and Radiative Transfer 132, 12 (2014).

[45] E. D. Palik, Handbook of optical constants of solids, Vol. 3 (Academic press, 1998).

[46] A. Narayanaswamy and G. Chen, Physical Review B 77, 075125 (2008).

[47] Y. Zheng and A. Ghanekar, Journal of Applied Physics 117, 064314 (2015).

[48] N. Vargaftik, B. Volkov, and L. Voljak, Journal of Physical and Chemical Reference Data 12, 817 (1983).

[49] L. Riedel, Chem.-Ing.-Tech 26, 259 (1954).

[50] V. P. Carey, Liquid-vapor phase-change phenomena (Hemisphere, New York, NY, 1992). 